\title{
Desenvolvimento de um manual ilustrado para o cirurgião-dentista da Unidade de Terapia Intensiva: relato de experiência
}

\author{
Development of an illustrated manual for the Intensive Care Unit surgeon- \\ dentist: experience report
}

\section{Desarrollo de un manual ilustrado para el cirujano-dentista de la Unidad de Terapia Intensiva: relato de experiencia}

Luana Taques ${ }^{1, a}$

luanataques@hotmail.com | https://orcid.org/oooo-0001-7738-7387

Paola Chrystine Machado Migdalsk ${ }^{1, a}$

paola chrystine@hotmail.com | https://orcid.org/o0oo-0002-7023-7498

Marcelo Carlos Bortoluzzi, $i^{1, b}$

mabortoluzzi@gmail.com | https://orcid.org/o000-0003-2756-5047

Eduardo Bauml Campagnoli,c

ebcampagnoli@hotmail.com | https://orcid.org/0000-0002-3413-028X

${ }^{1}$ Universidade Estadual de Ponta Grossa. Ponta Grossa, PR, Brasil.

${ }^{a}$ Residência Multiprofissional em Intensivismo pelo Hospital Universitário Regional dos Campos Gerais.

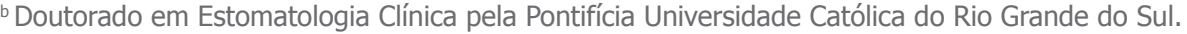

'Doutorado em Estomatopatologia pela Universidade Estadual de Campinas.

\section{Resumo}

Este relato objetiva apresentar o processo de desenvolvimento de um material escrito e ilustrado voltado para o dentista atuante em Unidades de Terapia intensiva, bem como os motivos e justificativas que levaram à decisão de sua necessidade. O cirurgião-dentista tem ampliado sua área de atuação e, por isso, necessita de materiais didáticos que forneçam uma base para essa nova forma de pensar a Odontologia, com um caráter integral e multiprofissional. Por não haver contato dos cirurgiões-dentistas com o ambiente hospitalar durante a graduação, salvo raras exceções, o material didático nesta área foi planejado para abranger desde os conhecimentos básicos até situações que envolvem maior conhecimento para a tomada de decisão. A metodologia utilizada foi o levantamento bibliográfico e a montagem de material escrito na forma de manual, além da produção e tratamento de fotografias ilustrativas, obtidas em um Hospital Universitário durante o processo de cuidado odontológico de pacientes críticos.

Palavras-chave: Comunicação interdisciplinar; Equipe hospitalar de odontologia; dicionários odontológicos como assunto; Materiais de ensino; Desenvolvimento de pessoal. 


\begin{abstract}
This report aims to present the process of developing a written and illustrated material for the dentist in intensive care units, as well as the reasons and justifications that led to the decision of their need. The dental surgeon has expanded his area of practice and, therefore, needs didactic materials that provide a basis for this new way of thinking about Dentistry, with an integral and multiprofessional character. Due to the lack of contact between dental surgeons and the hospital environment during graduation, with few exceptions, the teaching material in this area was designed to cover everything from basic knowledge to situations involving greater knowledge for decision making. The methodology used was the bibliographical survey and the assembly of written material in manual form, as well as the production and treatment of illustrative photographs obtained at a University Hospital during the dental care process of critical patients.
\end{abstract}

Keywords: Interdisciplinary communication; Hospital dental staff; Dental dictionaries as topic; Teaching materials; Staff development.

\title{
Resumen
}

Este relato tiene como objetivo presentar el proceso de desarrollo de un material escrito e ilustrado orientado hacia el dentista actuante en Unidades de Terapia intensiva, así como los motivos y justificaciones que llevaron a la decisión de su necesidad. El cirujano-dentista ha ampliado su área de actuación y, por eso, necesita materiales didácticos que proveen una base para esa nueva forma de pensar la Odontología, con un carácter integral y multiprofesional. Por no haber contacto de los cirujanos-dentistas con el ambiente hospitalario durante la graduación, salvo raras excepciones, el material didáctico en esta área fue planeado para abarcar desde los conocimientos básicos hasta situaciones que involucran mayor conocimiento para la toma de decisión. La metodología utilizada fue el levantamiento bibliográfico y el montaje de material escrito en la forma de manual, además de la producción y tratamiento de fotografías ilustrativas, obtenidas en un Hospital Universitario durante el proceso de cuidado odontológico de pacientes críticos.

Palabras clave: Comunicación interdisciplinaria; Personal de odontología en hospital; Diccionarios odontológicos como asunto; Materiales de enseñanza; Desarrollo de personal.

INFORMAÇÕES DO ARTIGO

\author{
Contribuição dos autores: \\ Concepção e desenho do estudo: Luana Taques \\ Aquisição, análise ou interpretação dos dados: Luana Taques e Paola Chrystine Machado Migdalski \\ Redação do manuscrito: Luana Taques e Paola Chrystine Machado Migdalski \\ Revisão crítica do conteúdo intelectual: Eduardo Bauml Campagnoli e Marcelo Carlos Bortoluzzi
}

Declaração de conflito de interesses: não há.

Fontes de financiamento: não houve.

Considerações éticas: para este relato de experiência, foram considerados os preceitos legais da resolução n0466/2012 e do Código de Ética em Odontologia, resolução n63/2005.

Agradecimentos/Contribuições adicionais: ao Hospital Universitário Regional dos Campos Gerais, por proporcionar a criação de um campo tão rico para a Odontologia da Universidade Estadual de Ponta Grossa.

Histórico do artigo: submetido: 23 jan. 2019 | aceito: 13 ago. 2019 | publicado: 20 dez. 2019.

Apresentação anterior: não houve.

Licença CC BY-NC atribuição não comercial. Com essa licença é permitido acessar, baixar (download), copiar, imprimir, compartilhar, reutilizar e distribuir os artigos, desde que para uso não comercial e com a citação da fonte, conferindo os devidos créditos de autoria e menção à Reciis. Nesses casos, nenhuma permissão é necessária por parte dos autores ou dos editores. 


\section{Introdução}

Ao longo dos últimos anos, a odontologia tem passado por mudanças bastante importantes. Antes vista como uma especialidade da saúde mais isolada, hoje é percebida pela comunidade científica como uma parte indissociável do cuidado à saúde integral, tendo campo de atuação inclusive dentro das Unidades de Terapia Intensiva (UTI). Isso se deve, em grande parte, a estudos que relacionaram a higiene oral como um fator importante para o desenvolvimento de pneumonia em pacientes restritos ao leito ${ }^{1,2}$.

Assim, o principal objetivo da presença do cirurgião-dentista (CD) na UTI é possibilitar a manutenção da higiene bucal dos pacientes críticos. É interessante observar que isso não envolve somente o procedimento descrito no Protocolo de Higiene Oral, embora essa seja a rotina diária do CD da UTI, mas também diz respeito a procedimentos um pouco mais invasivos - quando estes são necessários, liberados pela condição sistêmica e justificados pela intenção de manter a cavidade bucal livre de focos infecciosos. Desse modo, integrar a odontologia à equipe interdisciplinar é de extrema importância e tem por objetivo a conquista e o sustento da saúde oral, bem como orientações e assistência global ao paciente ${ }^{3}$.

Com a associação da importância da higiene oral efetiva na prevenção da pneumonia associada à ventilação mecânica (PAVM), vem se tornando cada vez mais evidente a relação custo-benefício entre infecções nosocomiais evitadas e a presença do cirurgião-dentista hospitalar ${ }^{4,5}$. Desse modo, cresce a procura por esse nicho de atuação em saúde e, consequentemente, desenvolve-se a ciência relacionada, de modo que, à medida que o tempo passa e experiências e pesquisas são acumuladas, permite-se o aprimoramento tanto do entendimento dos processos fisiopatológicos envolvidos nas alterações sistêmicas como dos protocolos a serem aplicados na assistência em saúde.

No Brasil, a formação de cirurgiões-dentistas competentes na atuação hospitalar e intensiva é, porém, uma ação que tem se mostrado bastante dificultada, principalmente pelo fato de não haver, na maioria dos cursos de graduação em Odontologia, o componente curricular Odontologia Hospitalar nos Planos político-pedagógicos (PPP) ${ }^{6}$. Há também o fato de não haver muitos materiais com abrangência de conteúdo hospitalar para consulta e desenvolvimento do processo de assistência odontológica em nível intensivo, o que justifica a criação desse tipo de instrumento didático, o qual os profissionais possam consultar como uma base para a resolução das dúvidas que venham a surgir no cotidiano do seu desenvolver laboral.

Destarte, o objetivo geral deste artigo foi relatar o processo de desenvolvimento de um material científicoliterário que possa vir a contribuir para o avanço da assistência odontológica ao paciente crítico, bem como para a formação de novos profissionais atuantes nesse crescente nicho profissional.

\section{Relato de experiência}

Este trabalho de produção literária foi resultado da experiência como residente de uma cirurgiãdentista especializanda em um Programa de Residência Multiprofissional em Intensivismo e a experiência de seus dois preceptores, associadas a um extenso levantamento bibliográfico que forneceu embasamento teórico para a explicação das rotinas e necessidades da assistência odontológica ao paciente crítico. O Programa de Residência Multiprofissional em Intensivismo aconteceu entre 2016 e 2018 no Hospital Universitário Regional dos Campos Gerais, vinculado à Universidade Estadual de Ponta Grossa, em Ponta Grossa, Paraná, Brasil.

O principal objetivo dos autores do material didático em questão foi compilar temas relacionados a aspectos práticos da atuação do cirurgião-dentista em ambiente hospitalar (com ênfase em Unidade de Terapia Intensiva) com o intuito de dar embasamento teórico para a tomada de decisão acerca dos principais problemas observados na rotina odontológica hospitalar. Além disso, os autores buscaram promover a inclusão do dentista nas equipes multiprofissionais, superando o isolamento profissional 
comumente observado nesta classe, e estimular a aquisição de competências pelos profissionais dentistas, mesmo aqueles não atuantes em ambiente hospitalar, no que se refere à avaliação integral do paciente.

O material didático foi elaborado em quatro fases: primeiro, a definição e arquitetura do conteúdo; segundo, o levantamento bibliográfico; terceiro, a produção das fotografias ilustrativas; e, em quarto e último lugar, o aprimoramento e a finalização.

FASE 1: A decisão pelos conteúdos contidos no manual foi baseada na experiência de dentistas atuantes em UTIs que não tiveram vivência hospitalar durante a graduação. Assim, reflete as dificuldades encontradas por este perfil de profissional para se enquadrar neste novo nicho de atuação que é a Odontologia Hospitalar, apresentando uma compilação de conhecimentos básicos necessários para a adaptação e o bom desenvolver do trabalho odontológico.

Os temas abordados no manual são apresentados no Quadro 1 a seguir, sendo cada categoria relacionada a um capítulo do material:

Quadro 1 - Temas abordados no material didático, divididos por capítulos

\begin{tabular}{|c|c|}
\hline Biossegurança no ambiente hospitalar & $\begin{array}{l}\text { Discute as precauções-padrão e as precauções pela rota de transmissão, } \\
\text { explicitando os isolamentos por contato, gotículas e aerossóis, e o } \\
\text { gerenciamento de resíduos em serviços de saúde. }\end{array}$ \\
\hline Equipamentos em UTI & $\begin{array}{l}\text { Apresenta os principais equipamentos utilizados na rotina de UTI, como } \\
\text { o monitor multiparamétrico, a bomba de infusão contínua, sistemas de } \\
\text { oxigenoterapia, ventilação não invasiva, ventilação mecânica, principais } \\
\text { dispositivos utilizados em UTI e o sistema de gases. }\end{array}$ \\
\hline $\begin{array}{l}\text { O que observar durante o atendimento } \\
\text { ao paciente crítico }\end{array}$ & $\begin{array}{l}\text { Discute o estado geral, o estado mental e o nível de consciência dos } \\
\text { pacientes críticos, bem como os sinais vitais, as restrições apresentadas } \\
\text { pelo doente e os sinais e sintomas agudos que podem ocorrer durante } \\
\text { o atendimento. }\end{array}$ \\
\hline Principais exames em UTI & $\begin{array}{l}\text { Capítulo em que são apresentados brevemente os principais exames } \\
\text { utilizados em UTI, laboratoriais e de imagem. }\end{array}$ \\
\hline $\begin{array}{l}\text { Pneumonia associada à ventilação } \\
\text { mecânica }\end{array}$ & $\begin{array}{l}\text { Apresenta e discute a fisiopatologia, os fatores de risco e os meios de } \\
\text { prevenção desta patologia intimamente relacionada à cavidade oral. }\end{array}$ \\
\hline Avaliação odontológica em UTI & $\begin{array}{l}\text { Explicita o que deve ser observado tanto intra quanto extrabucalmente } \\
\text { e qual a relação das alterações percebidas com a condição sistêmica do } \\
\text { paciente. }\end{array}$ \\
\hline Protocolo de higiene oral em UTI & $\begin{array}{l}\text { Apresenta protocolos de higiene oral tanto em pacientes intubados } \\
\text { quanto naqueles sob oxigenoterapia, bem como protocolo de } \\
\text { desinfecção e cuidados com próteses removíveis. }\end{array}$ \\
\hline $\begin{array}{l}\text { Manejo das principais alterações orais } \\
\text { observadas em pacientes críticos }\end{array}$ & $\begin{array}{l}\text { Incluindo ulcerações, crostas ressecadas e aderidas à mucosa, } \\
\text { sangramentos orais, entre outros. }\end{array}$ \\
\hline
\end{tabular}

Fonte: Os autores (2019).

A arquitetura do conteúdo foi pensada para que este seja utilizado como um manual, no qual se podem consultar tópicos referentes à prática odontológica hospitalar de rotina. A informação foi reunida em diferentes capítulos que abordam desde temas gerais e básicos como biossegurança em ambiente hospitalar até temas mais específicos como protocolos de higiene bucal em Unidade de Terapia Intensiva e pneumonia associada à ventilação mecânica. Cada capítulo tem suas referências indicadas separadamente, facilitando o entendimento e a busca por cada obra citada. 
Levantando as dificuldades enfrentadas durante essa primeira etapa do desenvolvimento do material, destaca-se a decisão pelos temas a serem pesquisados, haja vista que a percepção de cada profissional é subjetiva e pode levar a diferentes conclusões. Assim, apesar de julgados necessários e determinados estes oito principais tópicos para discussão, é evidente que muitos outros diferentes foram suscitados, não tendo sido incorporados ao material por questões de viabilidade.

FASE 2: O levantamento bibliográfico foi realizado por meio de pesquisa em periódicos e livros de diferentes áreas correlatas: medicina, fisioterapia, enfermagem e odontologia; além disso, foram consultados manuais de equipamentos, diretrizes institucionais e políticas públicas. Além da busca em bases de dados por meio de descritores controlados, foram revisadas as listas de referências dos artigos e dos livros selecionados. A pesquisa em periódicos deu-se pelo portal CAPES/MEC@, não havendo restrições de bases de dados; diferentes palavras-chave foram utilizadas para abranger todos os conteúdos programados. Em relação aos livros utilizados, foram consultados aqueles disponíveis na biblioteca da Universidade Estadual de Ponta Grossa, na biblioteca do Hospital Universitário Regional dos Campos Gerais e aqueles com acesso eletrônico liberado, além de alguns exemplares do acervo pessoal dos autores.

FASE 3: As ilustrações apresentadas são fotografias originais, tiradas dentro do Hospital Universitário Regional dos Campos Gerais. Foram fotografados equipamentos e dispositivos, bem como fácies e lesões labiais, peribucais e intrabucais de pacientes críticos. Os indivíduos que foram fotografados (ou seus responsáveis legais) assinaram o termo de consentimento livre e esclarecido permitindo o uso de sua imagem. Além disso, foi feita uma sequência de fotos demonstrando a correta adaptação da máscara N95, com a colaboração de uma enfermeira da equipe multiprofissional da UTI. Essa etapa da produção evidenciou bastante seu caráter interdisciplinar, pois diferentes profissionais se propuseram a auxiliar na obtenção de imagens de qualidade para o trabalho. O material resultante foi chamado de 'Manual Ilustrado para o cirurgião-dentista da Unidade de Terapia Intensiva', com o intuito de enfatizar suas ilustrações, que são em número de 80 .

FASE 4: o aprimoramento e a finalização do material compreenderam o tratamento das fotografias, com a colocação de tarjas para evitar a identificação dos pacientes e a edição das imagens, assim como a diagramação e organização do conteúdo escrito de forma a facilitar a aceitação e o entendimento do leitor. Além disso, foi realizada a conversão para o formato e-pub e a sua publicação em formato de livro digital ${ }^{10}$.

O conteúdo apresenta a descrição de conhecimentos básicos necessários para a formação do cirurgiãodentista atuante em UTI e serve como um roteiro que fornece informações fundamentais para a ambientação desse profissional de saúde que, por muito tempo, trabalhou em consultórios próprios, isolados de outras modalidades de assistência à saúde.

\section{Discussão}

As atividades desenvolvidas pelo CD hospitalar são complexas e exigem conhecimentos específicos que, na maior parte dos casos, não estão inseridos nos currículos dos cursos de graduação em Odontologia7 . Nesse sentido, existe a necessidade de oferta de cursos de habilitação para estes profissionais, visto que, durante a internação hospitalar há um aumento da demanda por assistência odontológica, seja ela cirúrgica ou clínica ${ }^{8}$. O Hospital Universitário Regional dos Campos Gerais, onde ocorreu o ensino em serviço que gerou a publicação do material em questão, não possui uma equipe de Odontologia Hospitalar no seu quadro permanente; desse modo, os Programas de Residência Multiprofissional vêm estruturando uma demanda até então inexistente. As residências multiprofissionais a nível hospitalar têm se revelado uma excelente 
opção para a formação de cirurgiões-dentistas atuantes nesta área, por se tratar de uma modalidade de pós-graduação lato sensu voltada para a educação em serviço e direcionada às profissões da área da saúde ${ }^{11}$.

A ausência da Odontologia Hospitalar como componente curricular dos planos político-pedagógicos (PPP) dos cursos de graduação é outro fator que dificulta o processo de capacitação de dentistas para a atuação hospitalar e intensiva. Um estudo exploratório quantitativo realizado por Lucas (2017) concluiu que 82\% das Instituições de Ensino Superior (IES) do Sul do Brasil não apresentam a Odontologia Hospitalar como componente curricular nos seus $\mathrm{PPP}^{6}$. Há, portanto, a necessidade de desenvolvimento de materiais-base que apresentem uma maior abrangência de conteúdo, que objetivem o entendimento das variáveis sistêmicas do paciente e do ambiente de atuação diferenciado, bem como da dificuldade e necessidade essencial de sua participação em equipes multiprofissionais de saúde. Por isso, é necessário que cirurgiões-dentistas expandam seu rol de instrução para que, mais que conhecer as variáveis sistêmicas, desenvolvam condição de julgamento criterioso sobre as comorbidades apresentadas pelo indivíduo, a fim de fornecer a melhor assistência em saúde, evitando causar qualquer tipo de dano ou prejuízo por negligência ou desconhecimento de causa. É preciso também que os profissionais tenham consciência de que as especialidades carecem de interligação para proporcionar uma assistência integrada que tenha como meta o bem-estar geral do indivíduo .

\section{Opção por material escrito de forma didática e objetiva}

A opção pela produção em formato de manual, com os temas explicados de forma simples e objetiva, deuse pela necessidade de união de temas que divergem muito entre si, mas que precisam ser compreendidos de maneira ampla para o bom desempenho profissional do dentista que atua na UTI. Pode-se perceber que o livro didático assume funções diferentes, dependendo da condição, do local e do momento em que é produzido e empregado nas diferentes situações ${ }^{9}$. Para Fiorentini e Moraes $^{12}$, a elaboração de um material didático deve conter mensagens motivadoras e inteligíveis, trazendo um equilíbrio entre os conhecimentos cotidiano e científico, entre os conteúdos transversais e disciplinares. Isso para que se considere o 'saber', o 'saber fazer', o 'saber ser' e o 'saber fazer junto', abrangendo o conhecimento dos fundamentos e conceitos, o domínio de habilidades, o desenvolvimento de atitudes e as interações sociais cooperativas ${ }^{12}$. O material em questão foi elaborado a partir desses requisitos, demonstrando, além da informação isolada, a visão do profissional que trabalha diariamente com pacientes críticos e as características da sua inserção na equipe multiprofissional.

\section{As ilustrações}

As ilustrações foram julgadas essenciais para alcançar o objetivo de levar conhecimento de forma sucinta para uso direto na prática profissional diária. Isso porque os dentistas, no geral, não têm contato com equipamentos, pacientes e processos característicos de unidades de terapia intensiva. Para Paulo Freire $^{13}$, a educação deve ocorrer a partir de estímulos obtidos no dia a dia dos educandos e, à medida que a aprendizagem ocorre, o universo desses alunos expande-se de forma paulatina. Assim, os estímulos visuais atendem perfeitamente às metas da educação, pois a percepção visual é a forma mais objetiva de interação do homem com o meio que o cerca ${ }^{13}$. Para Techy, o homem absorve a imagem, processa-a e, baseado em seus conhecimentos prévios, tira suas próprias conclusões ${ }^{14}$. De toda forma, a imagem, seja estática ou dinâmica, é uma forma muito comum de divulgação de informação, difundindo conhecimentos, desenvolvendo ideias e formando opiniões. Além disso, cresce progressivamente o uso da fotografia no processo ensino-aprendizagem; nas ciências, as ilustrações, as fotografias e os vídeos têm sido muito utilizados no processo científico e educacional ${ }^{15}$. 


\section{Escolha de cada tema}

É importante enfatizar que o processo de planejamento e de desenvolvimento de um material didático está relacionado de forma estreita com a concepção pedagógica do autor, tenha ele consciência ou não deste fato. Assim sendo, os temas selecionados foram respostas às dificuldades encontradas pelos autores durante o exercício odontológico em UTI.

A biossegurança foi alocada já no início do trabalho devido à sua importância fundamental na rotina profissional, e pelo fato de a biossegurança hospitalar ser bastante diferente daquela ensinada nos cursos de graduação em Odontologia. No hospital, devido às diferentes etiologias das enfermidades apresentadas pelos pacientes, há dois tipos de precauções: as Precauções Padrão e as Precauções Baseadas na Transmissão; estas últimas se dividem em três categorias, que são as precauções por contato, por gotículas e por aerossóis ${ }^{16}$. Em relação aos equipamentos da UTI, a necessidade foi relacionada ao desconhecimento dos cirurgiões-dentistas sobre o tema. Foram descritos de forma detalhada alguns aparatos como o monitor multiparamétrico, essencial para a monitorização do estado sistêmico do paciente; a bomba de infusão contínua; os diversos tubos utilizados, com suas mais variadas funções; e também os dispositivos utilizados na rotina, como as cânulas de traqueostomia e orofaríngeas, por exemplo.

A condição do paciente crítico deve ser observada antes e durante o atendimento odontológico. Foi esse o tema do terceiro capítulo, que discutiu o estado geral, o nível de consciência, os sinais vitais, as restrições apresentadas e as alterações agudas que podem ocorrer na presença do profissional. Os principais exames utilizados na rotina da UTI selecionados para inclusão no material envolveram os imaginológicos e alguns laboratoriais; a escolha foi baseada na rotina laboratorial observada nos prontuários dos pacientes críticos.

Outro tópico de discussão foi a pneumonia associada à ventilação mecânica (PAVM), enfermidade intimamente associada à condição bucal ${ }^{1}$. Como a PAVM é um forte fator que motivou a inserção da Odontologia nas UTIs, a decisão pelo seu detalhamento foi segura. A avaliação odontológica em pacientes críticos também foi especificada em um capítulo, considerando as principais diferenças observadas entre pacientes críticos e naqueles da clínica odontológica e, consequentemente, as dificuldades associadas.

O último capítulo tratou das principais lesões orais e periorais encontradas na rotina da terapia intensiva. As lesões compreendem, entre outras, ulcerações, acúmulo excessivo de biofilme, hipossalivação e saburra lingual ${ }^{17}$. $\mathrm{O} \mathrm{CD}$, porém, realiza não somente a parte curativa, mas atua como consultor agregado à equipe multidisciplinar ${ }^{18}$, dando apoio na assistência, otimizando o trabalho interdisciplinar e participando da tomada de decisão. Para tanto, o profissional dentista que atua em UTI deve estar preparado para discutir as principais demandas relacionadas ao sistema estomatognático em pacientes críticos, bem como as contribuições odontológicas possíveis nestas situações ${ }^{19}$.

\section{Decisão pela publicação em formato digital}

O livro é considerado um suporte de grande relevância na sociedade de informação. Após o surgimento das TIC (Tecnologias de Informação e Comunicação), apareceram, gradualmente, novos aparelhos de suporte à leitura, possibilitando a criação dos e-books. A conversão dos livros de papel para o formato digital permitiu a leitura em dispositivos tecnológicos; o surgimento dos e-books se situa quando da criação do Projeto Gutenberg, em 1971, que considera que o futuro dos livros são os livros digitais ${ }^{20}$. Chartier $^{21}$ afirma que presenciamos a terceira grande revolução da história do livro, chamada revolução digital.

Dentre as principais vantagens do $e$-book em relação ao livro físico destacam-se a agilidade de divulgação, o impacto ecológico, os custos e a facilidade de atualizações ${ }^{22}$. No caso do presente trabalho, a decisão pela publicação em formato de livro digital foi baseada na facilidade de divulgação e disseminação do arquivo, no baixo custo associado quando comparado ao livro físico e na rapidez do processo de publicação. 


\section{Considerações finais}

A produção do material didático para dentistas de Unidades de Terapia Intensiva enfatizou um componente que tem se mostrado cada vez mais importante em diferentes áreas, principalmente na educação e na saúde: a interdisciplinaridade. A compilação dos temas esclareceu a importância da formação em saúde para um atendimento integral do paciente, deixando claro que profissionais de saúde necessitam de saberes de áreas correlatas para um bom desenvolver do seu processo de trabalho.

Os escritos didáticos dão base à maioria das condições materiais para o processo ensino-aprendizagem e definem uma cultura legítima a ser transmitida. Embora tenha-se optado pelo desenvolvimento de material didático em forma de livro-manual, com conteúdo explicado e ilustrado, sabe-se que o livro determina o que será estudado e, para isso, seleciona conhecimentos válidos; porém, é necessário que o leitor busque aprofundamento nas áreas que lhe interessam por meio da pesquisa em diferentes fontes.

Espera-se que o material resultante da pesquisa realizada seja capaz de fornecer subsídio para a formação e atuação odontológica em Unidades de Terapia Intensiva. São necessários estudos para análise da aplicabilidade do material, da aceitabilidade pelos leitores e da necessidade de expansão de tópicos de pesquisa, visando uma maior abrangência de conteúdos.

\section{Referências}

1. Silva LD, Nepomuceno RM, Silva EF, Motta FP, Silva JA, Ferreira VG. Higiene oral ao paciente crítico: revisão de literatura. Rev Nursing. 2012;15(170):384-388.

2. Michels MA, Dick NRM, Zimerman RA, Malinsky RR. Auditoria em unidade de terapia intensiva: vigilância de procedimentos invasivos Rev Epidemiol Control Infect. 2013;3(1):12-16.

3. Ferreira JÁ, Londe LP, Miranda AF. A relevância do cirurgião-dentista na UTI: educação, prevenção e mínima intervenção. RCO. 2017;1(1):18-23.

4. Gaetti-Jardim Júnior E, Okamoto AC, Meca LB, Silva PP, Bombarda F, Schweitzer CM. Família Enterobacteriaceae, Acinetobacter baumannii e Pseudomonados na microbiota bucal de pacientes mantidos em unidades de terapia intensiva. Arch Health Invest. 2014; 3(4):40-8.

5. Morais TMN, Silva A, Avi ALRO, Souza PHR, Knobel E, Camargo LFA. A importância da atuação odontológica em pacientes internados em unidade de terapia intensiva. Rev. bras. ter. intensiva [Internet]. 2006 Dec [citado em 2019 set. 30];18(4):412-417. DOI: http://dx.doi.org/10.1590/S0103$\underline{507 \times 2006000400016 .}$

6. Lucas BB, Vieira JJLR, Besegato JF, Caldarelli PG. Ensino da Odontologia Hospitalar no Sul do Brasil. Revista da ABENO. 2017;17(2):68-75.

7. Associação Brasileira de Ensino Odontológico. $50^{\circ}$ Reunião da Associação Brasileira de Ensino Odontológico [Internet]. 2015 ago 12-14 [citado em 8 abr. 2019]; Salvador, Bahia. Disponível em: http://reuniaoanual.abeno.org.br/50reuniao/files/ementas/reuniao-sobep.pdf.

8. Godo APT, Francesco AR, Duarte A, Kemp, APT, Silva-Lovato CH. Odontologia hospitalar no Brasil. Uma visão geral. Rev Odontol UNESP. 2009;38(2):105-9.

9. Bittencourt CMF. Apresentação. Educ. Pesqui. [Internet]. 2004 [citado em 30 set. 2019]; 30(3), 471473. DOI: https://dx.doi.org/10.1590/S1517-97022004000300007.

10. Taques L, Bortoluzzi MC, Campagnoli EB. Manual ilustrado para o cirurgião-dentista da Unidade de Terapia Intensiva. 1ed. Porto Alegre: Revolução eBooks - Simplíssimo, 2018.

11. Brasil. Lei n. 11.129, de 30 de junho de 2005. Institui o Programa Nacional de Inclusão de Jovens ProJovem; cria o Conselho Nacional da Juventude - CNJ e a Secretaria Nacional de Juventude; altera as Leis no 10.683, de 28 de maio de 2003, e 10.429, de 24 de abril de 2002; e dá outras providências. Diário Oficial da União, Poder Executivo, Brasília, DF, 1 jul. 2005.

12. Fiorentini L, Morais R. Linguagens e interatividade na educação a distância. São Paulo: P\&D; 2000.

13. Freire P. Pedagogia do Oprimido. 42 ed. Rio de Janeiro: Paz e Terra; 2005. 
14. Techy A. A importância da fotografia na medicina. Rev. Bras. Reumatol. 2006;46(3):207-209.

15. Rosenblum N. A World History of Photography. New York: Abberville; 1997.

16. Aguiar DF, Lima ABG, Santos RB. Uso das precauções-padrão na assistência de enfermagem: um estudo retrospectivo. Rev Enfer Esc Anna Nery. 2008; 12(3):571-75.

17. Scannapieco FA, Rossa-Jr C. Doenças Periodontais versus Doenças Respiratórias. In: Brunetti MC. Periodontia Médica. São Paulo: SENAC; 2004. p. 391-409.

18. Morais TMN, Silva A, Avi ALRO, Souza PHRS, Knobel E, Camargo LFA. A importância da atuação odontológica em pacientes internados em Unidade de Terapia Intensiva. Rev Bras Ter Intensiva. 2006;18(4):412-17.

19. Terezakis E, Needleman I, Kumar N, Moles D, Agudo E. The Impact of hospitalization on oral health: a systematic review. J Clin Periodontol. 2011;38(7):628-36.

20. Figueiredo MMR. O papel dos e-books nas bibliotecas universitárias - o caso da Universidade de Aveiro [dissertação]. Portugal: Universidade de Coimbra; 2016.

21. Chartier R. A História ou a leitura do tempo. Belo Horizonte: Autêntica; 2009.

22. Espinoza N, Morales O. El texto eletronico:ċla desaparición de lo impreso o la aparición de una nueva fuente de lectura?. Lectura y Vida. 2002; 23(4). 